\title{
Penile Cancer by AJCC v8 Stage
}

National Cancer Institute

\section{Source}

National Cancer Institute. Penile Cancer by AJCC v8 Stage. NCI Thesaurus. Code C140075.

A term that refers to the staging of penile cancer according to the American Joint Committee on Cancer, 8th edition. This staging system applies to penile squamous cell carcinoma and associated histologic subtypes. It does not apply to urethral carcinomas, sarcomas, and melanomas. (from AJCC 8th Ed.) 